\title{
The phylogenetic relationships of insectivores with special reference to the lesser hedgehog tenrec as inferred from the complete sequence of their mitochondrial genome
}

\author{
Masato Nikaido ${ }^{1}$, Ying $\mathrm{Cao}^{2}$, Norihiro Okada ${ }^{1 *}$, and Masami Hasegawa ${ }^{2,3}$ \\ ${ }^{1}$ Graduate School of Bioscience and Biotechnology, Tokyo Institute of Technology, Yokohama, Japan \\ ${ }^{2}$ Institute of Statistical Mathematics, Tokyo, Japan \\ ${ }^{3}$ Department of Biosystems Science, Graduate University for Advanced Studies, \\ Hayama, Kanagawa, Japan
}

(Received 29 May 2002, accepted 6 December 2002)

\begin{abstract}
The complete mitochondrial genome of a lesser hedgehog tenrec Echinops telfairi was determined in this study. It is an endemic African insectivore that is found specifically in Madagascar. The tenrec's back is covered with hedgehoglike spines. Unlike other spiny mammals, such as spiny mice, spiny rats, spiny dormice and porcupines, lesser hedgehog tenrecs look amazingly like true hedgehogs (Erinaceidae). However, they are distinguished morphologically from hedgehogs by the absence of a jugal bone. We determined the complete sequence of the mitochondrial genome of a lesser hedgehog tenrec and analyzed the results phylogenetically to determine the relationships between the tenrec and other insectivores (moles, shrews and hedgehogs), as well as the relationships between the tenrec and endemic African mammals, classified as Afrotheria, that have recently been shown by molecular analysis to be close relatives of the tenrec. Our data confirmed the afrotherian status of the tenrec, and no direct relation was recovered between the tenrec and the hedgehog. Comparing our data with those of others, we found that within-species variations in the mitochondrial DNA of lesser hedgehog tenrecs appear to be the largest recognized to date among mammals, apart from orangutans, which might be interesting from the view point of evolutionary history of tenrecs on Madagascar.
\end{abstract}

Key words: Mitochondria, phylogeny, lesser hedgehog tenrec, Afrotheria, Eulipotyphla

The order Insectivora consists traditionally of six families: Talpidae (moles); Soricidae (shrews); Erinaceidae (hedgehogs); Solenodontidae (solenodons); Chrysochloridae (golden moles); and Tenrecidae (tenrecs; MacPhee and Novacek 1993). Recent molecular studies have, however, demonstrated that the order Insectivora is not monophyletic and that golden moles and tenrecs are not members of Insectivora but, instead, belong to a clade of endemic African mammals, called Afrotheria, that includes elephants, hyraxes, manatees, aardvarks, and elephant shrews (Stanhope et al. 1998a, 1998b). Other insectivores are often referred to as core-insectivores (Eulipotyphla), even though the position of hedgehogs

Edited by Fumio Tajima

* Corresponding author. E-mail: nokada@bio.titech.ac.jp

* Author to whom all correspondence should be addressed: N. Okada, Department of Biological Sciences, Graduate School of Bioscience and Biotechnology, Tokyo Institute of Technology, 4259 Nagatuta-cho, Midori-ku, Yokohama 226-8501, Japan remains to be clarified. Identification of the afrotherian clade, which includes tenrecs and golden moles, is one of the most important findings in molecular phylogenetics and, if the identification of this clade is valid, it has major implications with respect to mammalian evolution. The lesser hedgehog tenrec strongly resembles to true hedgehogs. The tenrec's back is covered by dozens of sharp spines and tenrecs often roll up, with spines erect, to protect themselves from predators. If these two taxa do not have a sibling relationship, the amazing resemblance of lesser hedgehog tenrecs to true hedgehogs represents convergent evolution that allowed tenrecs to fill a vacant ecological niche in Madagascar.

Analysis of the sequences of complete mitochondrial (mt) genomes is becoming a new standard for studies of inter-ordinal relationships among mammals (e.g., Penny and Hasegawa 1997). In the present study, we determined the complete sequence of the mtDNA of the lesser hedgehog tenrec (Echinops telfairi) in order to clarify the 
afrotherian status of tenrecs and examined its direct relation to hedgehogs.

A fresh sample of liver was obtained from the lesser hedgehog tenrec, and was preserved in 99\% ethanol. Total genomic DNA was isolated from this sample with phenol and chloroform (Blin and Stafford 1976). Extracted DNA was stored at $4^{\circ} \mathrm{C}$. The mtDNA of a lesser hedgehog tenrec was amplified by the polymerase chain reaction (PCR) with genomic DNA from a tenrec as template. The procedures for the amplification and sequencing of the mtDNA have been described in previous reports (Nikaido et al. 2000, 2001). The mitochondrial genome of the lesser hedgehog tenrec was fundamentally similar to those of other mammals, with a slight difference being an extended spacer region (35 nt) between genes for tRNA ${ }^{\mathrm{Tyr}}$ and subunit 1 of cytochrome $\mathrm{c}$ oxidase (COI). Moreover, heteroplasmy was observed not only with respect to numbers of repeat units but also with respect to the nucleotide sequence in the control region. The repeat unit in the control region was composed of six nucleotides, ATACGC.

Our analysis of complete sequences of mtDNA included sequences from the 43 species analyzed by Nikaido et al. (2001) plus the sequene of the mtDNA from $E$. telfairi (accession number; AB099484). We used the maximumlikelihood (ML) method for phylogenetic analysis (Felsenstein 1981; Kishino et al. 1990). The ProtML program in the MOLPHY package (ver. 2.3; Adachi and Hasegawa 1996b), the TREE-PUZZLE program (ver. 4.0.2; Strimmer and von Haeseler 1996) and the CodeML program in the PAML package (ver. 3; Yang 1997) were used to analyze the sequences of deduced mt-proteins with the mtREV-F model (Adachi and Hasegawa 1996a). The BaseML program in PAML was used to analyze the sequences of $12 \mathrm{~S}$ and $16 \mathrm{~S}$ rRNA, with the HKY85 model (Hasegawa et al. 1985). When we applied the TREEPUZZLE, CodeML and BaseML programs, we also used the discrete $\Gamma$-distribution model (with 8 categories) for site heterogeneity (Yang 1996). To evaluate the sum of the evidence obtained from the separate analyses of different genes (12 genes for mt-proteins and genes for $12 \mathrm{~S}$ rRNA and 16S rRNA), we applied the TotalML program in MOLPHY to the output files of the CodeML and BaseML programs (lfh files). The standard error (SE) of the log-likelihood difference between trees was estimated by the formula of Kishino and Hasegawa (1989). The platypus and two marsupials, namely, the opossum and wallaroo, were used as outgroups to root each eutherian tree.

With a large number of species, as in our data set (44 species), it is impossible to examine all the possible tree topologies by the ML method because of the explosive increase of the number of trees. The TREE-PUZZLE program provides an approximate method that partially overcomes this difficulty in searches for appropriate tree

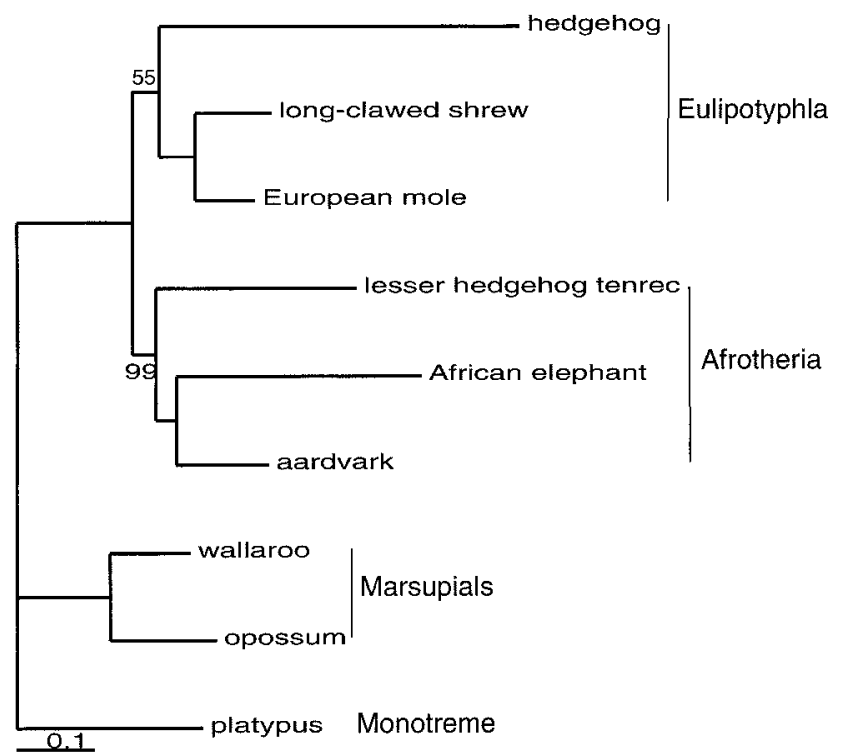

Fig. 1. A tree estimated by the TREE-PUZZLE program using the amino acid sequences of 12 proteins encoded by $\mathrm{mt}$ DNA. Horizontal lengths of branches are proportional to the estimated numbers of amino acid substitutions. Numbers at nodes refer to reliability values (percentages) of quartet puzzling.

topology. Figure 1 shows the results of TREE-PUZZLE analysis of the sequences of twelve deduced concatenated mt-proteins. In this tree, the tenrec is grouped with the elephant and the aardvark, forming the Afrotherian clade, eventhough the support value of $57 \%$ is not very high. Furthermore, the hedgehog is at a basal position in the eutherian tree (as is the same case with Krettek et al, 1995) and does not form a clade with the mole and shrew, however, support for this placement is also not very great (64\%). Recently the monophyly of afrotherians (including tenrecs) and the connection of hedgehogs to moles and shrews are well accepted by high bootstrap values in the analyses of nuclear genes (Murphy et al, 2001 a. b). There are big inconsistency between nuclear and our mitochondrial DNA analyses. So we re-examined these two issues, namely, the phylogenetic position of tenrecs and that of hedgehogs, more extensively, by using our complete mitochondrial genome data. Nikaido et al. (2001) compared the hedgehog-basal tree with the hedgehog-Eulipotyphla tree directly. They found that the former had a log-likelihood score that was almost indistinguishable from that of the latter when the $\Gamma$ model for site heterogeneity was applied. Taking the fact that the nuclear DNA analyses of Murphy et al. (2001 a, b) showed a strong connection of the hedgehogs to moles and shrews (not a basal), and that $\Gamma$ model is thought to be more realistic than simple evolutionary model, the hedgehog-basal tree might be an artifact due to an extremely rapid rate of nucleotide change (as is apparent in Fig. 1) and the biased amino acid composition 
of hedgehog mt-proteins. Indeed, the hedgehog is the only species among the 44 examined in Figure 1 in which the 12 concatenated mt-proteins have an amino acid composition that is significantly different from the average composition (chi-squared test; $P=1.4 \%$ ).

In order to examine phylogenetic relationships among the tenrec and relevant species more in detail, we also performed an exhaustive analysis with respect to the search for topology. However, such an analysis was impossible with the data from 44 species because of the huge number of possible trees. Therefore, we chose the following six species which were relevant to the issue of interest; tenrec, shrew, mole, hedghog, elephant, and aardvark. We used marsupials (wallaroo plus opossum) and a monotreme (platypus) as an outgroup. The shrew/ mole clade and the elephant/aardvark clade were established the previous analyses of mtDNA (Cao et al. 2000a; Nikaido et al. 2000, 2001) and, therefore, we accepted these clades in our subsequent analyses. Table 1 compares all 15 possible trees for tenrec, shrew/mole, hedgehog, elephant/aardvark and the outgroup. In the analysis that involved the $12 \mathrm{mt}$-proteins, the phylogenetic results depended somewhat on the model assumed in the ML analysis. The analysis of concatenated proteins without $\Gamma$ suggested Tree-2, with the tenrecAfrotheria and the hedgehog-basal relationships, while the analysis of concatenated proteins with the $\Gamma$ model suggested Tree-1 with the tenrec-Afrotheria and the hedgehog-Eulipotyphla relationships. However, the difference in log-likelihood between Tree-1 and Tree-2 was not significant (7.2 \pm 10.9$)$. Furthermore, analysis of the 12 individual proteins separately also suggested Tree-1 with the tenrec-Afrotheria and the hedgehog-Eulipotyphla relationships but again the difference in log-likelihood from Tree-2 was not significant $(4.7 \pm 12.3)$.

The introduction of the $\Gamma$ model for site heterogeneity improved the fitting of the model to the data in terms of AIC (Akaike 1974; Sakamoto et al. 1986; data not shown) and, furthermore, an analysis of separate proteins approximates the underlying evolutionary process better than the analysis of concatenated proteins, which does not explicitly assume heterogeneity of substitution pro- cesses across genes, and this conclusion holds even if site heterogeneity is taken into account with the $\Gamma$ model (Cao et al. 1999, 2000b; Adachi et al. 2000). Separate analysis with the model for each individual protein provided the best approximation of the data among the models that we considered. Analysis of both the 12 separate proteins and the 12 concatenated proteins with the $\Gamma$ model, suggested that Tree-1 is preferred. As mentioned previously, the preference for Tree- 2 when the ML method without $\Gamma$ was applied to the mt-proteins was probably an artifact due to an extremely rapid rate of nucleotide change and the biased amino acid composition of hedgehog mt-proteins. Thus, Tree-1 is probably the correct tree.

The subtotal of bootstrap proportions (BP) of Trees-1, -2 and -3 (tenrec-Afrotheria trees) amounted to $93.57 \%$. Table 1 also gives the total for the 12 mt-proteins $+12 \mathrm{~S}$ rRNA + 16S rRNA. The data for rRNA gave results consistent with those obtained with the proteins, and together the proteins and rRNAs gave subtotal of $99.27 \%$ for the BPs of the tenrec-Afrotheria tree (Fig. 2). It is noteworthy that a direct link between the apparently similar animals, the hedgehog and the tenrec (Trees-8, -9, and -12) was never recovered during a total of 10,000 bootstrap replications.

The results in Table 1 and Figure 2 suggest a closer relationship between the elephant and the aardvark than the elephant and the tenrec. Table 2 shows differences in log-likelihood scores for various relationships among these three Afrotherian species. Although Tree-A with the elephant/aardvark clade turned out to be the highest likelihood tree in total, other alternatives gave almost indistinguishable log-likelihood scores. Thus, more data are needed to resolve these relationships.

Our results suggest that the lesser hedgehog tenrec, an endemic African insectivore, is not closely related to the core-insectivores (eulipotyphlans) or to true hedgehogs. Thus, the non-monophyly of the insectivores is also supported by the complete mtDNA data, an observation that indicates the extent of difficulties associated with inferring common ancestries of these animals from morphological data. It is obvious that the amazing resemblance of

Table 1. Differences in log-likelihood scores $( \pm \mathrm{SE})$ for various relationships among E. telfairi (tenrec; abbreviation, Ech), Erinaceus europaeus (hedgehog; Eri), Sorex unguiculatus (shrew) + Talpa europaea (mole) (SorTal), and Loxodonta africana (elephant) + Orycteropus afer (aardvark) (LoxOry), with marsupials (wallaroo + opossum) and a monotreme (platypus) as the outgroup. The log-likelihood scores of the highest likelihood trees are given between $<$ and $>$ brackets. 'Concatenated' refers to the analysis of the concatenated sequences of 12 mt-proteins; 'separate' means that log-likelihood scores of 12 individual mt-proteins (with 12S rRNA and 16S rRNA as well) were estimated separately and were then summed by the TotalML program. RELL bootstrap proportions (Kishino et al. 1990) for 10,000 replications are given in parentheses for the total of the 12 mt-proteins + 12S rRNA + 16S rRNA.

\begin{tabular}{cccccc}
\hline \hline Tree & Mt-proteins & 12sr RNA & 16Sr RNA & Total & $($ BP) \\
\hline A ((Lox, Ory), Ech) & $<-28062.5>$ & $<-4686.9>$ & $-2.0 \pm 2.9$ & $<-37664.4>$ & $(0.4790)$ \\
B ((Ory, Ech), Lox $)$ & $-1.4 \pm 8.9$ & $-0.8 \pm 1.1$ & $<-4912.9>$ & $-0.2 \pm 8.1$ & $(0.4651)$ \\
C ((Lox, Ech), Ory) & $-6.0 \pm 8.0$ & $-1.0 \pm 0.8$ & $-2.4 \pm 2.6$ & $-7.3 \pm 7.8$ & $(0.0559)$ \\
\hline
\end{tabular}




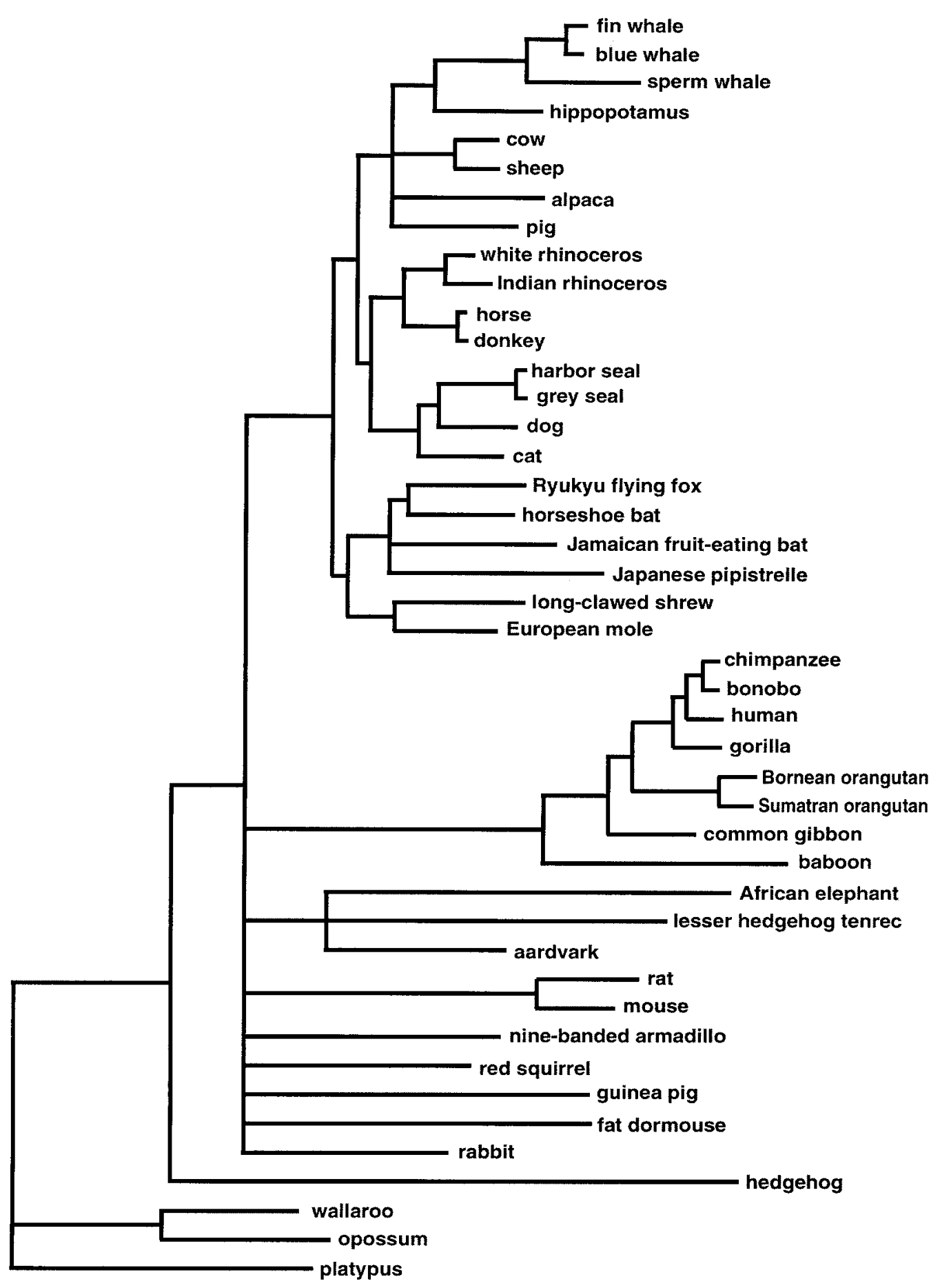

Fig. 2. The maximum-likelihood tree estimated for the nine species analyzed in Table 1 with the discrete $\Gamma$ model. Branch lengths were estimated by the CodeML program for the 12 concatenated mt-proteins. Numbers at nodes refer to bootstrap probabilities (percentages) from the sum of the separate analyses of the 12 mt-proteins + 12S rRNA + 16S rRNA shown in Table 1. The shrew/mole clade and the elephant/aardvark clade were assumed in this analysis. Marsupials and a monotreme were used as the outgroup.

tenrecs to hedgehogs is the result of convergent evolution, which might have been occurred in Madagascar to fill a vacant niche of the type occupied by hedgehogs in other places.
After completing this work, we learned that Mouchaty et al. (2000) had also sequenced the complete mt-genome of Echinops telfairi. Their analysis of mt-proteins also demonstrated the afrotherian status of tenrecs and our 
Table 2. Differences in log-likelihood scores ( \pm SE) for various relationships among E. telfairi (tenrec; abbreviation, Ech), L. africana (elephant; Lox), and O. afer (aardvark; Ory). The log-likelihood scores of the highest likelihood trees are given between < and $>$ brackets. 'Mt-proteins' indicates log-likelihood scores when values for 12 individual mt-proteins were estimated separately and then were summed by the TotalML programs. 'Total' indicates the total values from separate analyses of 12 individual proteins + 12S rRNA + 16S rRNA.

\begin{tabular}{|c|c|c|c|c|c|}
\hline \multirow{3}{*}{ Tree } & \multirow{2}{*}{\multicolumn{2}{|c|}{$\begin{array}{l}12 \mathrm{mt} \text {-proteins } \\
\text { Concatenated }\end{array}$}} & \multicolumn{3}{|c|}{ Proteins $+12 S+16 S$} \\
\hline & & & \multirow{2}{*}{$\begin{array}{c}\text { Separate } \\
\text { Without } \Gamma\end{array}$} & \multirow{2}{*}{$\begin{array}{c}\text { Separate } \\
\text { With } \Gamma\end{array}$} & \multirow[b]{2}{*}{ (BP) } \\
\hline & Without $\Gamma$ & With $\Gamma$ & & & \\
\hline 1 ((SolTal, Eri), (LoxOry, Ech)) & $-19.3 \pm 17.9$ & $<-28589.5>$ & $<-28062.5>$ & $<-37664.4>$ & $(0.5494)$ \\
\hline 2 ((SolTal, (LoxOry, Ech)), Eri) & $<-29882.4>$ & $-7.2 \pm 10.9$ & $-4.7 \pm 12.3$ & $-2.0 \pm 14.4$ & $(0.4431)$ \\
\hline 3 (SolTal, ((LoxOry, Ech), Eri) & $-45.7 \pm 14.2$ & $-19.2 \pm 8.4$ & $-17.8 \pm 9.3$ & $-24.6 \pm 10.3$ & $(0.0002)$ \\
\hline 4 (((SolTal, Eri), Ech), LoxOry) & $-55.0 \pm 24.4$ & $-17.0 \pm 8.2$ & $-20.7 \pm 10.8$ & $-33.4 \pm 13.1$ & $(0.0022)$ \\
\hline 5 (((SolTal, Eri), LoxOry), Ech) & $-47.5 \pm 24.2$ & $-17.9 \pm 7.9$ & $-19.8 \pm 10.8$ & $-33.5 \pm 12.8$ & $(0.0017)$ \\
\hline 6 (((SolTal, LoxOry), Ech), Eri) & $-18.3 \pm 18.0$ & $-24.0 \pm 15.5$ & $-24.6 \pm 18.2$ & $-40.2 \pm 21.4$ & $(0.0033)$ \\
\hline 7 (((SolTal, Ech), LoxOry), Eri) & $-43.0 \pm 14.3$ & $-31.6 \pm 14.1$ & $-34.6 \pm 16.7$ & $-49.9 \pm 20.1$ & $(0.0000)$ \\
\hline 8 ((SolTal, LoxOry), (Ech, Eri)) & $-44.8 \pm 25.4$ & $-33.2 \pm 14.6$ & $-33.0 \pm 17.0$ & $-57.1 \pm 20.0$ & $(0.0000)$ \\
\hline 9 (SolTal, (LoxOry, (Ech, Eri))) & $-54.5 \pm 22.7$ & $-31.6 \pm 12.2$ & $-30.6 \pm 13.9$ & $-57.5 \pm 16.8$ & $(0.0000)$ \\
\hline 10 (((SolTal, LoxOry), Eri), Ech) & $-62.6 \pm 23.9$ & $-35.5 \pm 14.3$ & $-37.1 \pm 16.6$ & $-61.3 \pm 19.6$ & $(0.0001)$ \\
\hline 11 (SolTal, ((LoxOry, Eri), Ech)) & $-83.8 \pm 20.1$ & $-34.8 \pm 11.7$ & $-34.4 \pm 13.5$ & $-61.9 \pm 16.4$ & $(0.0000)$ \\
\hline 12 ((SolTal, (Ech, Eri)), LoxOry) & $-66.3 \pm 25.3$ & $-38.9 \pm 13.0$ & $-41.5 \pm 15.1$ & $-63.4 \pm 17.9$ & $(0.0000)$ \\
\hline 13 (((SolTal, Ech), Eri), (LoxOry) & $-92.3 \pm 22.8$ & $-42.8 \pm 12.3$ & $-44.9 \pm 15.0$ & $-66.5 \pm 17.7$ & $(0.0000)$ \\
\hline 14 ((SolTal, (LoxOry, Eri)), Ech) & $-92.2 \pm 22.6$ & $-45.6 \pm 12.3$ & $-46.2 \pm 15.2$ & $-73.7 \pm 17.9$ & $(0.0000)$ \\
\hline 15 ((SolTal, Ech), (LoxOry, Eri)) & $-100.6 \pm 21.4$ & $-46.7 \pm 12.2$ & $-50.2 \pm 14.8$ & $-76.9 \pm 17.7$ & $(0.0000)$ \\
\hline
\end{tabular}

results are consistent with theirs. The results of Mouchaty et al. differ, however, from ours in that, in their analysis, the three lipotyphlan taxa, tenrecs (Tenrecidae), moles (Talpidae) and hedgehogs (Erinaceidae), are dispersed on the eutherian tree, while Erinaceidae represents a basal eutherian lineage. Such conclusion are not in harmony with the results of recent extensive analyses of nuclear genes and partial mt-genes by Murphy et al. (2001) and Madsen et al. (2001), who placed hedgehogs in Eulipotyphla. In our best ML model, hedgehogs are situated in the eulipotyphlan lineage. The apparent conflict between the conclusions of Mouchaty et al. (2000) and those of Murphy et al. (2001 a, b) and Madsen et al. (2001) appears to be the result of an artifact introduced by the extremely rapid rate of nucleotide change and biased amino acid composition of hedgehog mt-proteins.

When we compared our tenrec sequence with that reported by Mouchaty et al. (2000), we found that two sequences differed by as many as 42 amino acid residues (among 3,393 residues in the 12 proteins used in our phylogenetic analysis) even though the two sequences were from the same species. This difference is the largest of the within-species variations among mammalian mitochondria observed to date, with the exception of the 152 differences detected between Bornean (database accession number, D38115) and Sumatran (X97707) orangutans. Reported within-species of amino acid differences in the mt-proteins of other species are as follows: 18 in human (African D38112 vs. European X93334); 9 in chimpanzee (D38113 vs. X93335); 18 in gorilla (D38114 vs. X93347); 10 in pig (AJ002189 vs. AF034253); and 7 in mouse (J01420 vs. AB042809). The 42 differences in $E$. telfairi are similar to the 46 differences between horse and donkey in terms of absolute numbers (X79547 vs. X97337). We used the method of Thorne et al. (1998) to estimate branching dates without assuming a molecular clock, with the same calibration and constraints as those used by Nikaido et al. (2001), and estimated the timing of the divergence of the two individual tenrecs examined as $3.3 \pm 0.8 \mathrm{MyrBP}$. It will be interesting in the future to investigate the ecological and biogeographical factors in Madagascar that might have produced such a large within-species variation in tenrec mtDNA.

This work was supported by research grants from the Ministry of Education, Science, Sports and Culture of Japan to N. O. and M. H., and from the Graduate University for Advanced Studies (Group Research to M. H.).

\section{REFERENCES}

Adachi, J., and Hasegawa, M. (1996a) Model of amino acid substitution in proteins encoded by mitochondrial DNA. J. Mol. Evol. 42, 459-468.

Adachi, J., and Hasegawa, M. (1996b) MOLPHY: Programs for Molecular Phylogenetics ver. 2.3, Computer Science Monographs, No. 28. Institute of Statistical Mathematics, Tokyo Adachi, J., Waddell, P., Martin, W., and Hasegawa, M. (2000) 
Plastid genome phylogeny and a model of amino acid substitution for proteins encoded by chloroplast DNA. J. Mol. Evol. 50, 348-358.

Akaike, H. (1974) A new look at the statistical model identification. I. E. E. E. Trans. Autom. Contr. A. C. 19, 716-723.

Blin, N., Stafford, D. W. (1976). A general method for isolation of high molecular weight DNA from eukaryotes. Nucleic Acids Res. 3, 2303-2308.

Cao, Y., Kim, K., Ha, J., and Hasegawa, M. (1999) Model dependence of the phylogenetic inference: Relationship among carnivores, perissodactyls and cetartiodactyls as inferred from mitochondrial genome sequences. Genes Genet. Syst. 74, 211-217.

Cao, Y., Fujiwara, M., Nikaido, M., Okada, N., and Hasegawa, M. (2000a) Interordinal relationships and time-scale of eutherian evolution as inferred from mitochondrial genome data. Gene 259, 149-158.

Cao, Y., Sorenson, M. D., Kumazawa, Y., Mindell, D. P., and Hasegawa, M. (2000b) Position of turtles in amniotes: Total evidence of molecular analyses. Gene 259, 139-148.

Hasegawa, M., Kishino, H., Yano, T. (1985) Dating of the human-ape splitting by a molecular clock of mitochondrial DNA. J. Mol. Evol. 22, 160-174.

Kishino, H., and Hasegawa, M. (1989) Evaluation of the maximum likelihood estimate of the evolutionary tree topologies from DNA sequence data, and the branching order in Hominoidea. J. Mol. Evol. 29, 170-179.

Kishino, H., Miyata, T., Hasegawa, M. (1990) Maximum likelihood inference of protein phylogeny and the origin of chloroplasts. J. Mol. Evol. 30, 151-160.

Krettek, A., Gullberg, A., and Arnason, U. (1995) Sequence analysis of the complete mitochondrial DNA molecule of the hedgehog, Erinaceus europaeus, and the phylogenetic position of the Lipotyphla. J. Mol. Evol. 41, 952-957.

MacPhee, R. D. E., and Novacek, M. J. (1993) Definition and relationships of Lipotyphla. In: Mammalian Phylogeny: Placentals. (eds.: F. S. Szalay, M. J. Novacek, and M. C. McKenna), pp. 13-31. Springer-Verlag, New York.

Madsen, O., Scally, M., Douady, C. J., Kao, D. J., Debry, R. W., Adkins, R., Amrine, H. M., Stanhope, M. J., de Jong, W. W., and Springer, M. S. (2001) Parallel adaptive radiations in two major clades of placental mammals. Nature 409, 610 614.

Mouchaty, S. K., Gullberg, A., Janke, A., and Arnason, U. (2000) Phylogenetic position of the Tenrecs (Mammalia: Tenrecidae) of Madagascar based on analysis of the complete mitochondrial genome sequence of Echinops telfairi. Zool. Scripta. 29, 307-317.

Murphy, W. J., Eizirik, E., Johnson, W. E, Zhang, Y. P., Ryder, O. A., and O'Brien, S. J. (2001a) Molecular phylogenetics and the origins of placental mammals. Nature 409, 614618.

Murphy, W. J., Eizirik, E., O’Brien, S. J., Madsen, O., Scally, M., Douady, C. J., Teeling, E., Ryder, O. A., Stanhope, M. J., de Jong, W. W., and Springer, M. S. (2001b). Resolution of the early placental mammalian radiation using Bayesian phylogenetics. Science 294, 2348-2351.

Nikaido, M., Harada, M., Cao, Y., Hasegawa, M., and Okada, N. (2000) Monophyletic origin of the order Chiroptera and its phylogenetic position among Mammalia inferred from the complete mitochondrial DNA sequence of a Japanese megabat, Ryukyu flying fox (Pteropus dasymallus). J. Mol. Evol. 51, 318-328.

Nikaido, M., Kawai, K., Cao, Y., Harada, M., Okada, N., and Hasegawa, M. (2001) Maximum likelihood analysis of the complete mitochondrial genomes of eutherians and a reevaluation of the phylogeny of bats and insectivores. J. Mol. Evol. 53, 508-516.

Penny, D., and Hasegawa, M. (1997) Molecular systematics: the platypus put in its place. Nature 387, 549-550.

Sakamoto, Y., Ishiguro, M., and Kitagawa, G. (1986) Akaike Information Criterion Statistics. D. Reidel. Publ. Comp., Dordrecht.

Stanhope, M., Madsen, O., Waddell, V. G., Cleven, G. C., de Jong, W. W., and Springer, M. S. (1998a) Highly congruent molecular support for a diverse superordinal clade of endemic African mammals. Mol. Phyl. Evol. 9, 501-508.

Stanhope, M., Waddell, V. G., Madsen, O., de Jong, W. W., Hedges, S., Cleven, G. C., Kao, D., and Springer, M. S. (1998b) Molecular evidence for multiple origins of Insectivora and for a new order of endemic African insectivore mammals. Proc. Natl. Acad. Sci. U. S. A. 95, 9967-9972.

Strimmer, K., and von Haeseler, A. (1996) Quartet puzzling: a quartet maximum-likelihood method for reconstructing tree topologies. Mol. Biol. Evol. 13, 964-969.

Thorne, J., Kishino, H., Painter, I. (1998) Estimating the rate of evolution of the rate of molecular evolution. Mol. Biol. Evol. 15, 1647-1657.

Yang, Z. (1996) Among-site rate variation and its impact on phylogenetic analyses. Trends Ecol. Evol. 11, 367-372.

Yang, Z. (1997) PAML: a program package for phylogenetic analysis by maximum likelihood. CABIOS 13, 555-556. 